\title{
Concomitant Medication End Relative to Reference Time Point
}

National Cancer Institute

\section{Source}

National Cancer Institute. Concomitant Medication End Relative to Reference Time Point. NCl Thesaurus. Code C87862.

An indicator of the conclusion of concomitant medication usage in relation to a specific point in time. 